\title{
LA POLÍTICA DE FOMENTO A LA FORMACIÓN DE DOCTORES Y LA DOCENCIA UNIVERSITARIA EN ARGENTINA: ALGUNAS TENSIONES NO RESUELTAS
}

\author{
THE POLICY TO PROMOTE PHD'S AND THE UNIVERSITY TEACHING IN ARGENTINA: \\ SOME UNRESOLVED TENSIONS
}

Martín Unzué ${ }^{1}$

\section{RESUMEN}

El presente trabajo aborda el problema de la baja inserción de doctores en la docencia universitaria argentina actual. Como resultado de un proceso de consolidación tardío de los doctorados, en particular en algunas disciplinas, la demanda de esa formación no había sido, tradicionalmente, un elemento de peso en la selección de los docentes universitarios argentinos. Con el fuerte crecimiento de los programas doctorales, que se verifica desde comienzos del presente siglo, y el importante aumento del número de doctores, el proceso de selección de los docentes universitarios ha ido modificándose paulatinamente, casi podríamos decir que por presión generacional. A pesar de ello, las universidades siguen mostrando niveles muy bajos de formación doctoral en sus planteles de profesores, lo que constituye una anomalía.

PALABRAS CLAVE: Doctores. Universidad. Profesores universitarios.

\begin{abstract}
The present paper addresses the problem of the low insertion of PhD's in the Argentine university teaching. As a result of a process of late consolidation of doctorates, particularly in some disciplines, the demand for such training had not traditionally been a major factor in the selection of Argentine university professors. With the strong growth of doctoral programs, which has taken place since the beginning of this century, and the significant increase in the number of doctors, the selection process of university professors has gradually changed. Despite this, universities continue to show very low levels of doctoral training in their faculty establishments, which is an anomaly.
\end{abstract}

KEYWORDS: PhD. University. Professors.

\section{INTRODUCCIÓN}

El desarrollo de los doctorados en Argentina ha conocido un fuerte proceso de aceleración en la primera década del presente siglo, aunque no con igual intensidad en todas las ramas disciplinarias. Las razones son múltiples.

\footnotetext{
${ }^{1}$ Doctor en Ciencias Sociales, economista. Profesor e Investigador en el Instituto de Investigaciones Gino Germani, Universidad de Buenos Aires. E-mail: unzuemart@ yahoo.com - ORCID: http://orcid.org/0000-00022369-7563
}

Submetido em: 07-03-2017 / Aceito em: 06-04-2017 / Publicado em: 10-04-2017.

\begin{tabular}{|l|l|l|l|l|l|l|} 
(C) Rev. Inter. Educ. Sup. & Campinas, SP & v.3 & n.1 & p.150-166 & jan./abr. 2017 & ISSN 2446-9424 \\
\hline
\end{tabular}


En primer lugar, los posgrados en general, particularmente las especializaciones y maestrías, crecen fuertemente desde la década de 1990, tomando más impulso luego de la sanción de la llamada Ley de Educación Superior (LES), en el año 1995.

No nos detendremos sobre ese proceso, que ha sido ampliamente estudiado (BARSKY, 1997; LVOVICH, 2009).

La LES, que sigue regulando el sistema en la actualidad, generará un marco normativo favorable al desarrollo de los posgrados, que podrán ser una fuente de ingresos adicionales para las universidades, en particular en medio de las penurias presupuestarias de esos años, así como un complemento salarial a los docentes más renombrados.

Sin embargo el análisis del perfil de los posgrados que se desarrollan en esa década deja en claro el fuerte predominio de los de carácter profesionalista. Se trata fundamentalmente de especializaciones, cursos de actualización y algunas maestrías, que en áreas disciplinares con campos profesionales amplios, como sucede en el derecho, las ciencias médicas y las ciencias económicas, se vuelven rápidamente espacios con gran aceptación.

Ese crecimiento inicial de los posgrados no se verifica en los doctorados, que seguirán percibidos como espacios de formación académica, de investigadores, no profesionales, y por lo tanto, más alejados de las demandas de actualización o reconversión laboral que dieron difusión a los posgrados profesionalistas citados.

Sin embargo, luego de la crisis política y económica de los años 2001-2002 en Argentina, y con el proceso de reordenamiento primero, y luego de incremento de los presupuestos orientados al sector de ciencia y tecnología, que se verifica desde el año 2004, se producirá un importante cambio en el escenario que tendrá un claro impacto en los doctorados.

El objetivo de este trabajo será analizar las transformaciones que se han suscitado en el sistema de posgrados y en el sistema universitario argentino en el período 2003-2015, con especial interés por el desarrollo de los doctorados. En ese sentido, y sobre la base de la compilación de datos estadísticos de diversas fuentes, y la elaboración propia de los datos faltantes en las estadísticas nacionales, el presente trabajo avanzará sobre la cuantificación de ese proceso de crecimiento de los doctorados, analizando su impacto en el sistema universitario argentino, y particularmente entre los profesores universitarios.

Se buscará analizar los éxitos y las limitaciones de esas transformaciones, señalando el rol de la docencia universitaria en su interacción con los doctorados, así como cuantificar el desarrollo de ese vínculo, señalando la tradicional anomalía de la universidad argentina, que se ha consolidado sobre la ausencia del requisito de ser doctor graduado para acceder a los cargos docentes.

\section{EL CRECIMIENTO DE LOS DOCTORADOS EN ARGENTINA}

Si bien en las disciplinas tradicionales, como la medicina o las ciencias exactas, la carrera científica ya estaba estructurada con la incorporación de los doctorados, lo mismo no 
pasaba en otros campos del saber, donde ese nivel irrumpe tardíamente (DE LA FARE Y LENZ, 2012; UNZUÉ, 2011).

En esas áreas la carrera académica o de investigación había logrado, salvo excepciones puntuales de profesores con doctorados en universidades europeas 0 norteamericanas, prescindir de esos posgrados, en muchos casos inexistentes o de desarrollo muy escaso en el país.

A este proceso, se le sumó también la interrupción de carreras académicas producto de los efectos de la inestabilidad institucional argentina sobre las universidades, desde el golpe de estado de 1966, pasando por la llamada "Noche de los bastones largos" y fundamentalmente el período que se abre con el nuevo golpe de estado de 1976 y se extiende hasta 1983. Recordemos que en ese ciclo, purgas, expulsiones, exilios y desapariciones de personal académico significarán en muchos casos, la interrupción de carreras universitarias y de investigación, al menos desde la formalidad institucional y con particular énfasis en algunas áreas disciplinares como las ciencias sociales y las humanidades.

El caso de las ciencias sociales es emblemático, aunque no único, de un campo de saberes que no verá su desarrollo e institucionalización completa hasta el retorno a la democracia en los años 80, cuando comienza su lento proceso de consolidación. En él, se comenzará con la apertura de carreras de grado, y de facultades, que tendrán muy buena acogida por parte de los potenciales estudiantes, dado que el número de estudiantes universitarios del área se vuelve rápidamente mucho mayor al de varias carreras científicas y tecnológicas.

Pero este desarrollo inicial no se da en el nivel de los posgrados y particularmente de los doctorados, hasta que la consolidación de las disciplinas, casi dos décadas después, ya sea un dato concreto.

El resultado será una baja institucionalización de algunos campos de saberes, en particular de aquellos que fueron vistos como más "conflictivos" durante los períodos de gobiernos autoritarios, y por lo tanto los que recibieron la mayor atención y represión en esos ciclos, lo que se traducirá por carreras académicas en muchos casos informales o incompletas a la luz de los estándares consolidados internacionalmente.

Las políticas de CyT implementadas a partir de la asunción de un nuevo gobierno, en el año 2003, tomarán como eje la inversión en formación de investigadores, asociando la misma, en consonancia con la experiencia internacional, con la tarea de los doctorados. Ello dinamizará los programas de becas doctorales en el país produciendo una serie de transformaciones relevantes en el funcionamiento del sistema universitario y en el de CyT.

Debemos notar al menos tres cuestiones sobre este punto. La primera, es que a diferencia de otras experiencias regionales, la decisión de invertir en CyT, y la de orientar esa inversión a la formación de doctores, también se completó con la disposición de favorecer su formación en la Argentina, lo que resolvía entre otras cosas el ya clásico problema del no siempre claro retorno de los investigadores que realizan sus estadías en países centrales.

En segundo lugar, que ese modo de dinamizar la formación doctoral introdujo un elemento novedoso para la tradición del sistema universitario argentino: las becas de

\begin{tabular}{l|l|l|l|l|l|l} 
() Rev. Inter. Educ. Sup. & Campinas, SP & v.3 & n.1 & p.150-166 & jan./abr. 2017 & ISSN 2446-9424 \\
\hline
\end{tabular}


dedicación exclusiva que supusieron que en el nivel del doctorado una parte sustancial de los estudiantes tengan una dedicación a tiempo completo a la universidad.

Finalmente, la combinación de becas numerosas, para hacer doctorados en el país, generará un fuerte proceso de crecimiento de los propios programas doctorales, así como de la oferta de cursos, del número de doctorandos, y rápidamente, ya desde el año 2007-2008, en el número de doctores graduados en la Argentina.

Como se puede apreciar en el Grafico 1 que está a continuación, el número de estudiantes de doctorado en el país pasa de un número entre 3500 y 4000 a comienzos de siglo a casi 26.000 en 2015 .

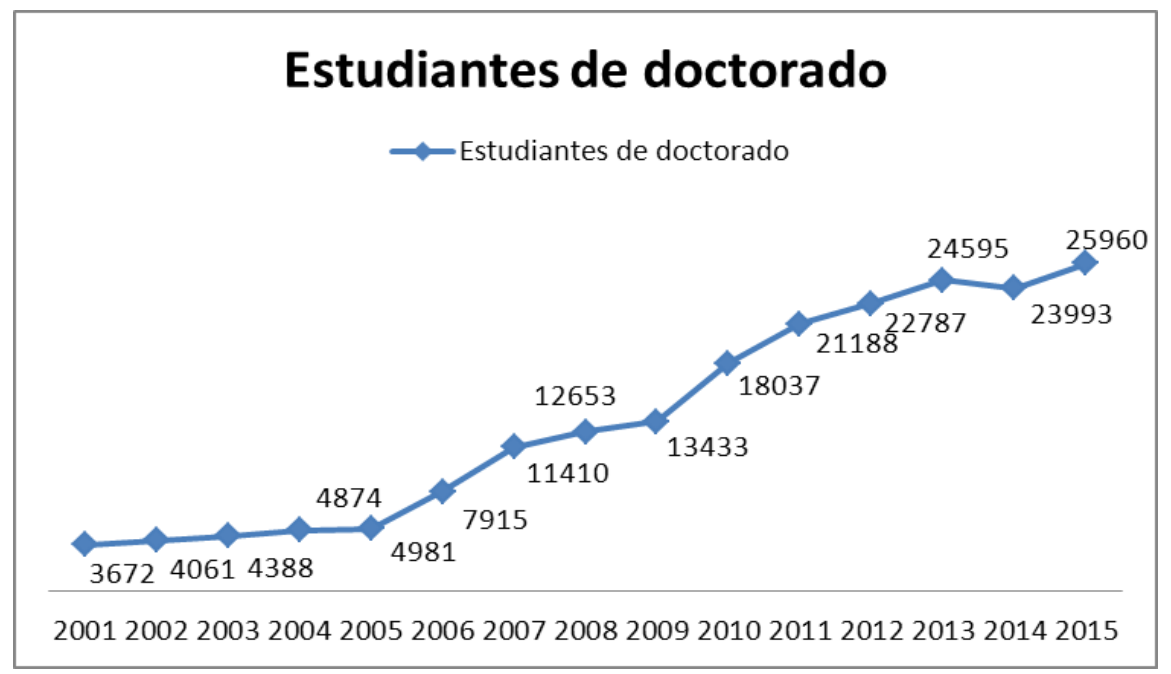

Grafico 1. Estudiantes de doctorado en Argentina 2001-2015. Fuente: Elaboración propia en base a datos de la SPU

\section{EL FINANCIAMIENTO DE LAS BECAS DOCTORALES}

Es cierto que no todo ese crecimiento se explica por el aumento de la oferta de becas, pero sí se puede ver que las mismas crecen muy significativamente en el período, y que producen un efecto sobre el todo el sistema de posgrados, favoreciendo su desarrollo.

La principal fuente de este financiamiento de la formación de nuevos doctores fue el CONICET (Consejo Nacional de Investigaciones Científicas y Técnicas), que incrementó sustancialmente su programa de becas doctorales, reuniendo un número próximo al $60 \%$ del total de las becas del sistema.

A eso se le deben agregar las becas doctorales que otorga la Agencia Nacional de Promoción Científica y Tecnológica (ANPCyT) vía el FONCyT, que son becas asociadas a proyectos de investigación financiados por esta agencia.

En el siguiente cuadro podemos ver cómo se produce, particularmente en el período 2003-2005, un fuerte crecimiento de la oferta de becas de ambos organismos, que más que se duplica, de estar por debajo de las 1500 a superar las 3000 anuales. 


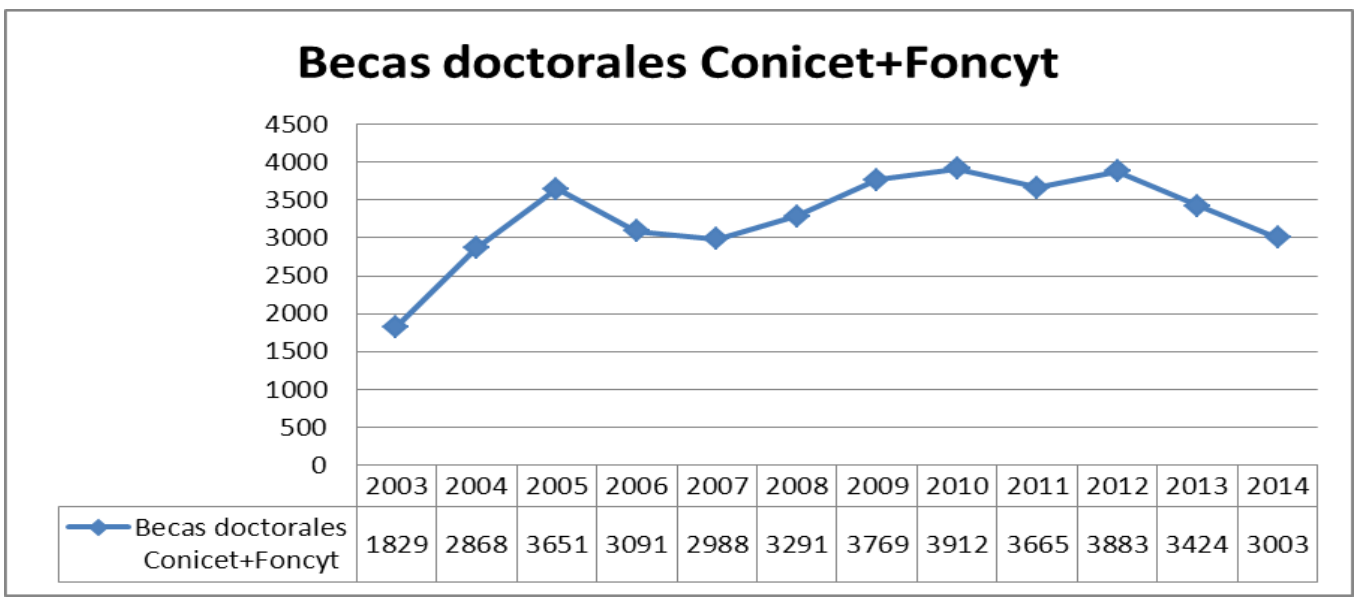

Grafico 2. Becas doctorales otorgadas por CONICET y ANPCyT vía FONCyT (2003-2014)

Fuente: Elaboración propia en base a datos de ambos organismos

En este período también tendremos un proceso de crecimiento, aunque mucho más modesto, de becas doctorales ofertadas por el propio sistema universitario, pero que contribuirán a generar un crecimiento de la oferta de becas del sistema en el decenio 20032013. A pesar de ello, no hay información centralizada sobre estas becas.

Esta nueva situación tendrá como efecto una importante reorientación de las carreras académicas y docentes en las universidades argentinas, aunque desigual en su incidencia por disciplina.

El doctorado comenzará a ser visto como un paso necesario sea para el ingreso a la Carrera de Investigador Científico en el CONICET (CIC), o, para optimizar las posibilidades de inserción y desarrollo laboral en el sistema universitario, algo que como veremos sucederá parcialmente.

Pero además, la disponibilidad de becas resultará una salida fácil (y prometedora) para salvar el complejo tránsito de la universidad al mercado laboral, en particular en algunas disciplinas con baja demanda profesional y alto número de estudiantes graduados.

La posibilidad real de acceder al vigorizado sistema de becas despertará múltiples vocaciones "científicas" en disciplinas en las que, previamente, esa opción de desarrollo académico parecía marginal.

Hay un dato adicional que debe ser destacado. Una debilidad histórica del sistema universitario argentino, muy vinculada con sus formas de ingreso directo y su gratuidad, es la baja tasa de graduación de sus estudiantes. Mucho se ha trabajado e investigado sobre este punto. La novedad que introduce la proliferación de becas de nivel doctoral es que revierte, al menos en ese nivel de estudios, esa tradicional falencia. Los datos de terminalidad de tesis de los becarios resultan altos.

A modo de ejemplo, la información que suministra el CONICET analizando el período 1998-2015 muestra que el $80 \%$ de los becarios doctorales han concluido sus estudios y defendido sus tesis. Ese número es del $60 \%$ al quinto año de la beca, lo que supone el cumplimiento perfecto de los plazos de los doctorados en Argentina, y llega al $80 \%$ en el

\begin{tabular}{|l|l|l|l|l|l|l} 
(C) Rev. Inter. Educ. Sup. & Campinas, SP & v.3 & n.1 & p.150-166 & jan./abr. 2017 & ISSN 2446-9424 \\
\hline
\end{tabular}


caso de los que alcanzan el título dentro de los tres años posteriores a la finalización de la misma (es decir, en un período de 8 años desde el inicio del doctorado).

Entre los años 2003 y 2015 el número de tesis doctorales defendidas por becarios del CONICET aumentó un 398\%, pasando de 234 a 1165 tesis (CONICET, 2016).

El análisis por área muestra que son las más tradicionales las que presentan los mejores niveles de terminalidad y cumplimiento de los plazos de las becas. En este sentido, en el área de las Ciencias Exactas y Naturales se alcanza el 75,8\% de defensas de tesis al finalizar la beca (quinto año), en Ciencias Biológicas y Salud el número es de 71,2\%, en el área de Tecnología el 66,9\%, en el área de Agrarias e Ingeniería el 63,4\% y en Ciencias Sociales y Humanidades, que presenta el número más bajo, el $45 \%$, aunque este número estaría creciendo en las últimas cohortes (CONICET, 2016).

El resultado de esto es, que el fuerte aumento del número de becas doctorales a partir de los años 2003-2005, se traducirá en un crecimiento sustancial del número de doctores graduados en la Argentina que se verifica desde los años 2008-2009.

Si tomamos los datos que presentamos en el Grafico 3, podemos ver que los becarios del CONICET defienden un número ligeramente superior a las 200 becas anuales al comienzo del siglo, y pasan a estabilizarse en un número en torno a las 1200 una década después. El crecimiento más pronunciado lo encontramos en el período 2007-2010.

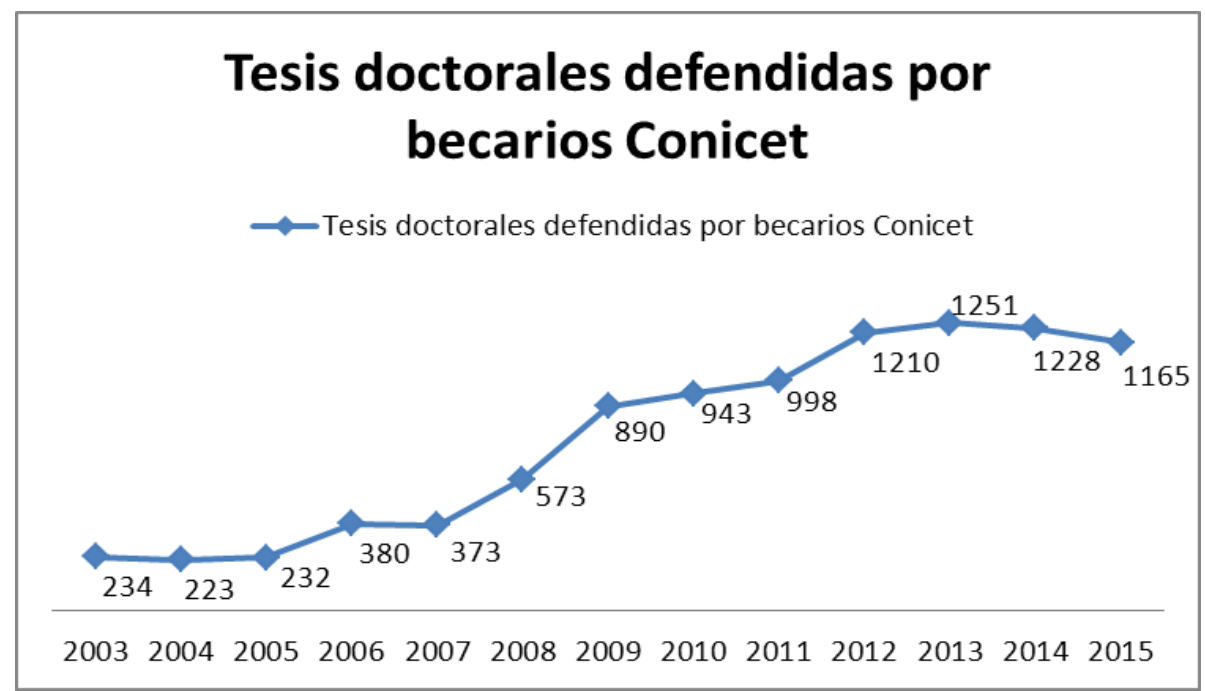

GRAFICO 3: Tesis defendidas por becarios Conicet 2003-2015

Fuente: Elaboración propia en base a datos del CONICET

Es difícil reconstruir el número total de doctores que se gradúan por año, en particular porque la principal universidad del país, la de Buenos Aires, que se estima que reúne al $40 \%$ de los estudiantes de este nivel, no está incluida en los datos agregados del sistema en en buena parte del período $2003-2015^{2}$.

\footnotetext{
${ }^{2}$ Las versiones sobre esta ausencia son diversas, pero estaría vinculada a la noción "fuerte" de autonomía que tienen algunas universidades nacionales como la de Buenos Aires, y a cuestiones de manejo presupuestario interno. 
La cantidad de graduados doctores sin contar a la UBA, en base a la información relevada por la Secretaría de Políticas Universitarias del Ministerio de Educación de la Nación, sería para el período 2007-2011 la que agregamos en el siguiente grafico.

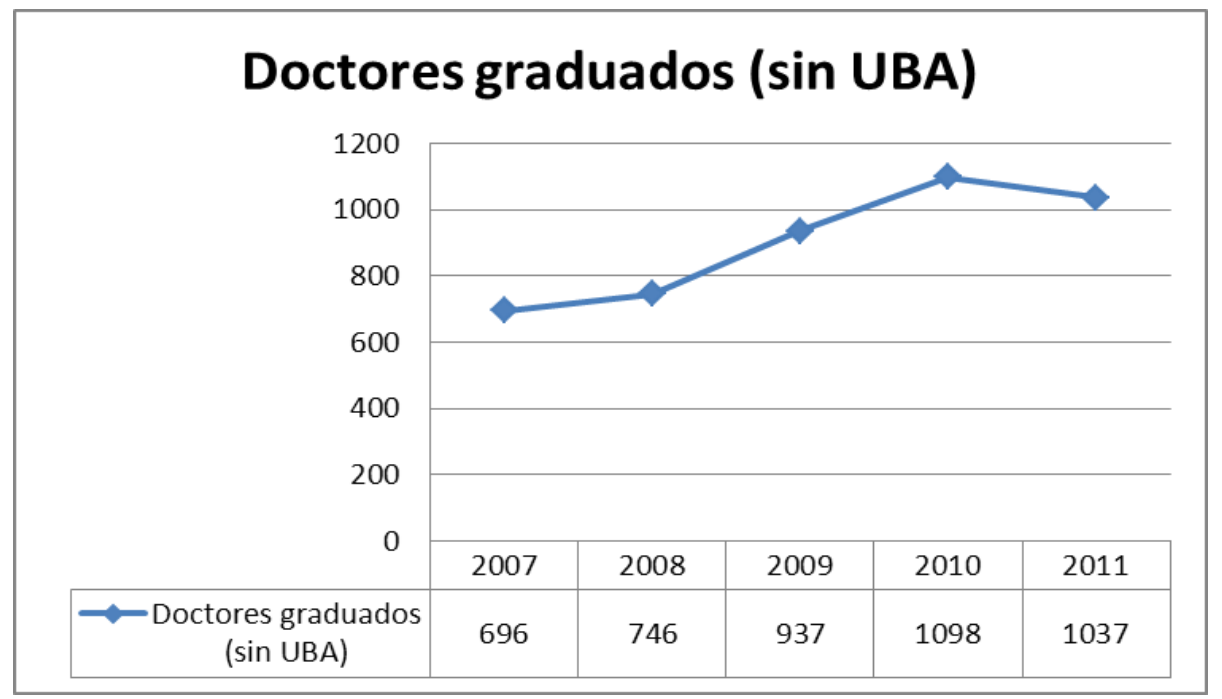

Grafico 4. Doctores graduados en Argentina sin UBA (2007-2011) Fuente: Elaboración propia en base a datos SPU

Esto nos permite ver que, incluso sin los datos de la UBA, el número de doctores graduados se incrementa casi un 50\% en sólo 5 años.

Hemos completado los datos de la SPU con los datos que surgen de los departamentos de posgrado de las principales facultades de la Universidad de Buenos Aires, lo que nos permite hacer una nueva estimación de los números de doctores graduados en Argentina en todo el período.

En primer lugar hay que notar que el comportamiento de las facultades, como el de las disciplinas, es muy variado. Tenemos campos de saberes donde hay una verdadera explosión de los doctorados en el período, mientras en otros el crecimiento es mucho más moderado.

El siguiente cuadro analiza el número de doctores formados en la Universidad de Buenos Aires por áreas disciplinares.

Lo primero que podemos ver es que hay perfiles de carreras muy diferentes, algo sin dudas vinculado con el número de graduados de cada facultad, pero también con la tradición profesionalista o académica que predomina en cada disciplina.

Podemos encontrar áreas muy numerosas en el grado pero con poca propensión a la actividad académica. En estos casos los doctorados parecen relativamente estables en el tiempo o ligeramente ascendentes en el número de doctores que forman, pero esa estabilización se da en números bajos, o incluso muy bajos si lo cruzáramos con los de egresados del grado como vemos en Derecho, Ciencias Económicas o Arquitectura.

Otro perfil lo vemos en las áreas con escasa cantidad de graduados del grado, pero fuerte crecimiento del doctorado. Esto lo vemos por ejemplo en Agronomía, Ciencias 
Veterinarias o Ingeniería. Los doctorados de estas áreas han crecido, pero se encuentran con la limitación del bajo número de aspirantes.

El tercer perfil es el de las Ciencias Sociales (similar al de las Humanidades), que se destacan por tener un buen nivel de egresados del grado, y un vertiginoso crecimiento del doctorado, que en consecuencia, alcanza niveles de formación muy relevantes en número.

Finalmente, un cuarto perfil lo encontramos en el área de Farmacia y Bioquímica, que también muestra un importante aumento del número de doctores formados, aunque partiendo de números más elevados al comienzo del ciclo.

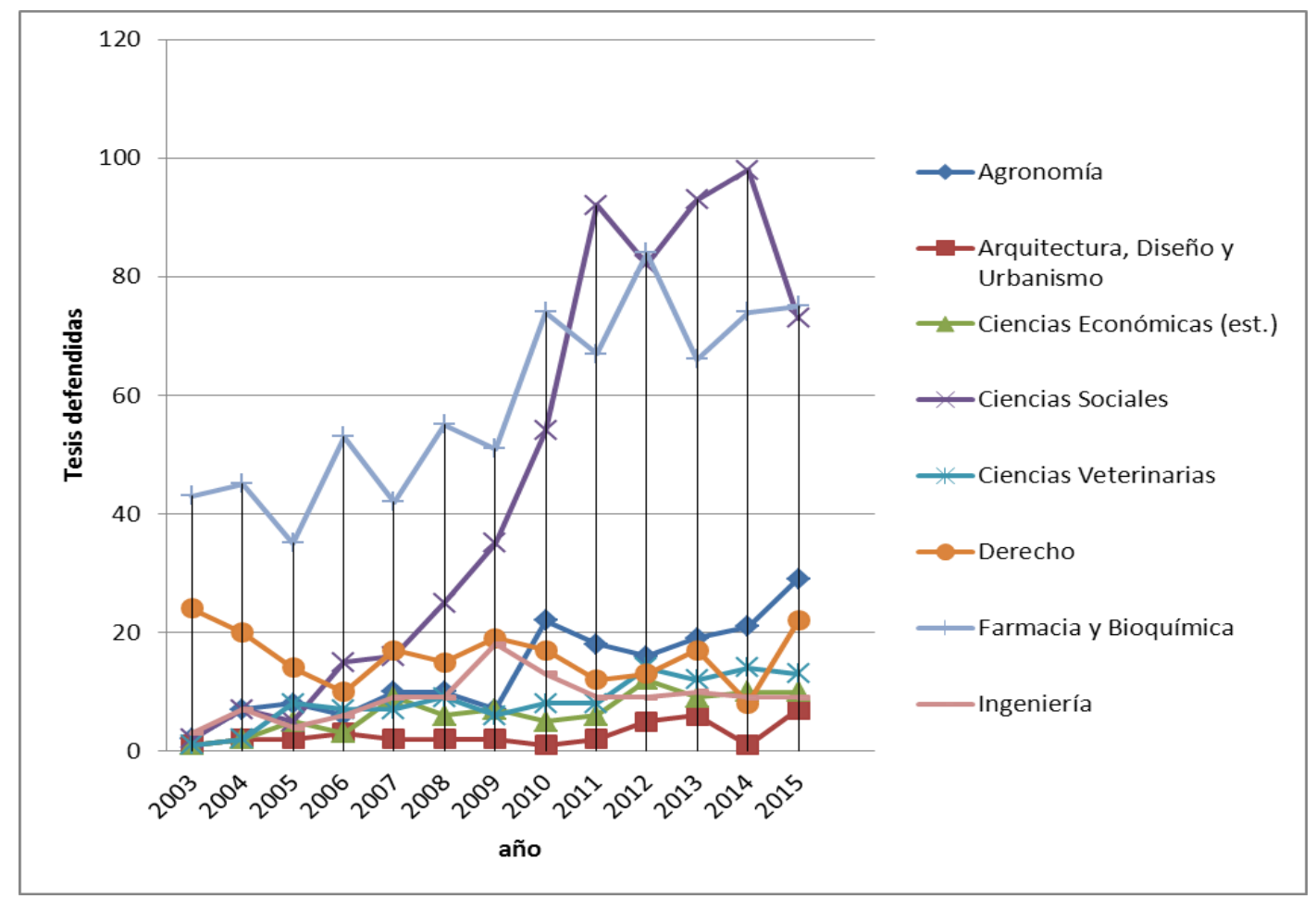

Grafico 5. Tesis Doctorales Defendidas en Facultades de la UBA (2003-2015) Fuente: Elaboración propia en base a datos provistos por las Facultades a excepción de Ciencias Económicas (estimado)

Si nos centramos en el número de doctores que produce la UBA, podemos completar el Grafico 4 para corroborar ese crecimiento del sistema, que supera el 100\% en el sexenio. 


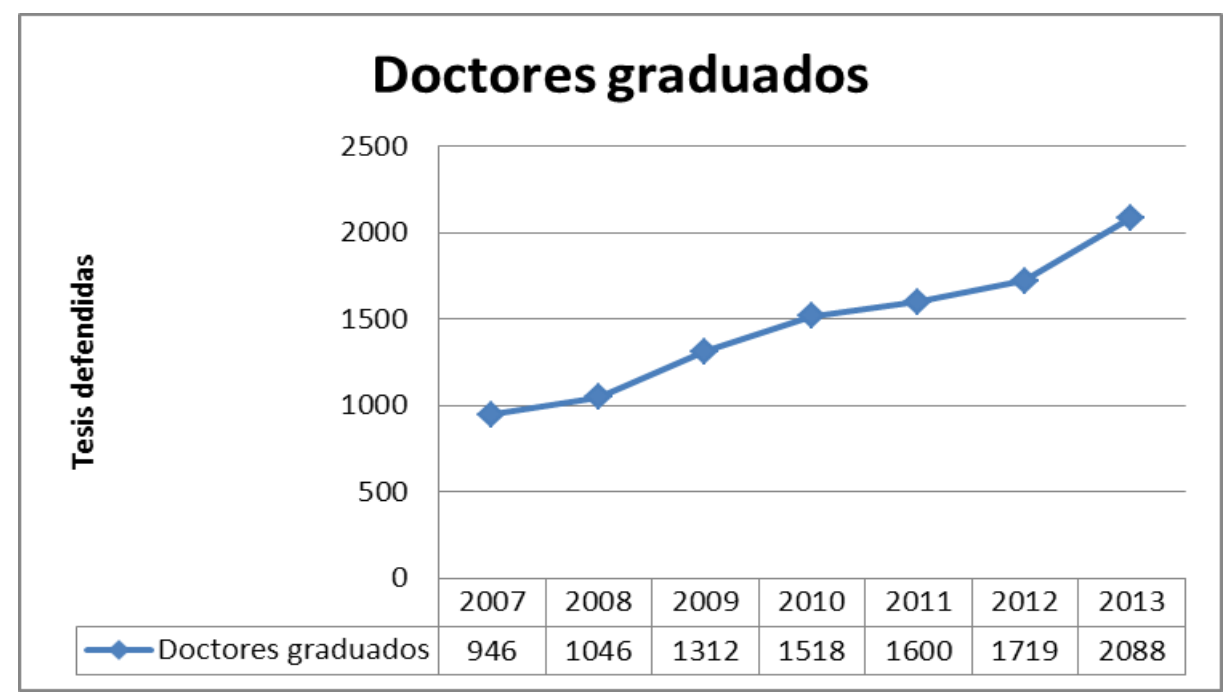

Grafico 6. Doctores graduados en Argentina con estimación UBA (2007-2013) Elaboración propia en base a datos SPU y Facultades UBA

Otro dato a considerar, es que el relativamente tardío desarrollo de los posgrados en varios campos disciplinares, produjo como efecto esperable, la convivencia de diversas generaciones realizando el doctorado, y una edad promedio de cursada y graduación bastante elevada en términos internacionales.

Sobre este punto, el análisis de los datos del Doctorado en Ciencias Sociales de la Universidad de Buenos Aires, sin duda muy emblemático en ese aspecto por ser un doctorado relativamente nuevo, en una facultad también de creación reciente (es producto de la normalización democrática en 1987), y por ello clara expresión de las dificultades para consolidar a las disciplinas sociales en la Argentina, pero además, porque se trata del doctorado más numeroso del país en el área, muestra que la graduación se concentra en los estudiantes de entre 30 y 35 años $(47,6 \%)$ seguido por la categoría 36-40 años con 22,8\%, y que tan sólo un $3 \%$ se graduaba con menos de 30 años, lo que representa un porcentaje similar al correspondiente con los franjas de 56-60 años y 61-65.

Al igual que lo relevado por CONICET para sus becarios, los datos del Doctorado en Ciencias Sociales de la UBA muestran que el 53\% de los graduados lo hacen en 5 o 6 años, y que el número sube al $90 \%$ si se considera hasta 9 años.

Que el comportamiento sea asimilable al de los becarios se explica porque en este caso, como en el de buena parte de los doctorados que más han crecido, el 66,55\% de sus estudiantes disponen de becas.

En cuanto a la progresión del número de doctores graduados de la que ya dimos cuenta en el grafico 5, podemos ver que es muy vertiginosa. Se inicia con una cantidad insignificante entre 2002-2003 (3 doctores), sube a 12 en 2004-2005, 31 en 2006-2007, 61 en 2008-2009, 149 en 2010-2011, 177 en 2012-2013 y se reduce ligeramente a 140 en 20142015. Esta trayectoria acelerada no es exclusiva de las Ciencias Sociales.

En términos generales podemos afirmar que hay un gran crecimiento del número de doctores graduados en el ciclo 2003-2015, pero que no es homogéneo en todas las disciplinas.

\begin{tabular}{l|l|l|l|l|l|l|} 
(C) Rev. Inter. Educ. Sup. & Campinas, SP & v.3 & n.1 & p.150-166 & jan./abr. 2017 & ISSN 2446-9424 \\
\hline
\end{tabular}


Otra variable que podríamos agregar, es la distribución geográfica de ese crecimiento, que nos muestra que hay ciertas regiones centrales que son las absolutas responsables de ese desarrollo. Tomando como ejemplo los doctores graduados en el año 2011 podemos ver que el Área Metropolitana Buenos Aires (AMBA) explica el 49\% de todos los doctores graduados en la Argentina, seguida por la Provincia de Córdoba con el 14\%, la Provincia de Buenos Aires con el $13 \%$ y la de Santa Fe con el $11 \%$. Esos cuatro distritos, sin dudas los más poblados, los que concentran el mayor número de universidades, explican el $87 \%$ del total de nuevos doctores de ese año, mientras que las otras regiones se ven muy marginadas del proceso de desarrollo de los doctorados, por diversas razones, entre ellas las dificultades para abrir doctorados.

\section{LA INSERCIÓN LABORAL DE LOS DOCTORES Y LA DOCENCIA UNIVERSITARIA}

El incremento del número de doctores de reciente formación en Argentina, producto de las políticas de expansión del sector sostenidas en el período 2004-2015, permitió, en un primer momento, la incorporación de nuevos investigadores a algunos organismos científicos nacionales.

El caso del CONICET es el más emblemático, en parte porque fue el principal financiador de las becas, y por ende, una parte sustancial de sus becarios doctorales ingresaron a la Carrera de Investigador Científico.

Sin embargo, en torno al año 2010, comenzó a vislumbrarse que el sendero de crecimiento del organismo tenía ciertos cuellos de botella. Problemas de inversión en infraestructura, saturación de algunas líneas de trabajo, dificultades administrativas en el organismo para digerir un proceso de fuerte y acelerado crecimiento.

Por ello, y en coincidencia con el despliegue de nuevas orientaciones en la política de $\mathrm{CyT}$, a partir de ese momento empiezan a introducirse diversos limitantes al ingreso de los nuevos doctores a la carrera, buscando federalizar los recursos, e introducir cierta prioridad por ejes temáticos.

Esto planteó una serie de problemas en torno al destino de los nuevos doctores que no lograban insertarse en la investigación básica tradicionalmente asociada al CONICET, o que quedaban marginados sea por la zona geográfica de procedencia (la política de federalización de recursos humanos que implementa el organismo desde ese momento comienza a desalentar a los doctores de las áreas de mayor desarrollo) o por los temas de trabajo, asociados parcialmente a las áreas disciplinares.

La debilidad de la inversión en CyT del sector privado argentino, un fenómeno compartido con buena parte de los países de la región, también significó la dificultad de lograr insertar investigadores en el mismo, salvo algunos nichos muy específicos pero poco significativos en números.

De este modo, las universidades nacionales que como vimos, compartieron el esfuerzo de la formación de los doctores, y que se vieron beneficiadas indirectamente por el 
crecimiento de esos posgrados, quedaron en la mira, como posibles espacios de inserción laboral de los mismos en un escenario que comenzaba a mostrar ciertos desajustes.

\section{DOCENTES DOCTORES EN UNIVERSIDADES ARGENTINAS, AÚN UNA DEUDA PENDIENTE}

El sistema de educación superior argentino es sin dudas muy amplio y diverso y ha evolucionado significativamente en los último años.

A diferencia de otros países de la región, es el sub-sistema universitario el que ocupa el lugar central en la educación superior, concentrando recursos, instituciones, y estudiantes.

Ese sistema universitario también se presenta como muy heterogéneo. Compuesto por 53 universidades públicas federales que albergan casi a tres cuartas partes de la matrícula estudiantil, además de concentrar la mayor parte de la investigación académica, el lugar de las 49 universidades privadas, 21 institutos universitarios y 6 universidades provinciales resulta bastante menor frente a las primeras.

A pesar de esas diferencias por tipo de institución, que también se encuentran atravesadas por realidad disciplinar, en términos generales podemos decir que la docencia universitaria argentina se ha caracterizado por el predominio de cargos con dedicaciones parciales, en disciplinas predominantemente profesionalistas, lo que supone que la mayor parte de los docentes ejercen sus respetivas profesiones en ámbitos (públicos o privados) ajenos a la universidad e incluso que eso ha sido valorado como un elemento deseable: los saberes de la práctica profesional, que se transmiten a los estudiantes, más allá de las teorías.

Las consecuencias de ello han sido numerosas. Desde los niveles salariales generalmente bajos, a las escasas cargas de investigación o extensión universitaria para la mayor parte de los docentes, consolidan formas de carrera académica que tienden a desmarcarse de la tradicional estructura de las universidades de otras regiones.

El punto sobre el que queremos avanzar en este artículo es la escasa participación de los doctores en el universo de los docentes universitarios argentinos.

\section{PROFESORES Y DOCTORES}

La ya citada Ley de Educación Superior hace pocas consideraciones sobre la relevancia de los doctorados en el reclutamiento de profesores universitarios.

Esto es entendible por lo presentado más arriba: cuando se sanciona la ley, en 1995, el grado desarrollo de los doctorados argentinos en muchas disciplinas hacía impensable ir mucho más allá en esa exigencia y el problema se volvía aún más grave en las áreas geográficas más relegadas.

Por eso la LES se limita a una fórmula muy vaga para referirse a la formación necesaria para el ejercicio de la docencia universitaria como la del artículo 36 donde dice: "gradualmente se tenderá a que el título máximo sea una condición para acceder a la 
categoría de profesor universitario". Notemos que no se hace referencia a los doctorados, sino al "título máximo".

Pero esa ambigüedad ha sido muy poco operativa. A fin de cuentas, la ley no fija plazos ni obligaciones, y por ende, las universidades no se han concentrado en la tarea de reclutar doctores para sus cuerpos docentes a pesar del fuerte crecimiento de los mismos en la última década.

Este punto resulta una anomalía si consideramos que el principal destino laboral de los doctores en la mayor parte de las experiencias internacionales es insertarse como investigadores en las universidades, e incluso que es poco frecuente que se acceda a la docencia universitaria sin poseer la credencial de doctor.

Sin embargo, en las universidades argentinas el título de doctor no ha sido, y en ciertos espacios sigue sin ser necesariamente, un elemento determinante para el ingreso al cuerpo de docentes y de docentes-investigadores.

Sobre este punto podemos hacer tres consideraciones:

- La poca relevancia que le asignan las formas de evaluación de las carreras de grado a la existencia de docentes con título de doctor, supone una ausencia de estímulo para que las mismas recluten doctores.

- En segundo lugar, los reglamentos de concursos docentes, en especial los de profesores, que deberían ser el medio habitual de acceso a la docencia, suelen no hacer referencia directa a los títulos de doctor como requisito para acceder a los cargos. La fórmula "antecedentes equivalentes" sigue siendo muy utilizada en estos casos, incluso más a la hora de evaluar profesores que auxiliares docentes.

- En tercer lugar, el modo en que se ha producido el desarrollo de los doctorados, concentrado regionalmente en las áreas de mayor densidad socio-productiva, colisiona con las dos olas de creación de universidades nacionales que se han dado en los años 90 y desde 2003 en adelante, que han permitido la apertura de numerosas universidades en regiones en las que no existían doctores para integrar los cuerpos docentes en formación.

Como consecuencia, un análisis de los datos producidos por la Secretaría de Políticas Universitarias muestra que sólo una pequeña minoría los docentes universitarios argentinos poseen título de doctor, y que esto se reproduce en todo el escalafón y con todas las dedicaciones al cargo, a pesar de que se verifica un incremento en el período 2009-2013.

Como podemos ver en el siguiente cuadro, con los datos disponibles, y es importante notar que recién a partir de 2009 las estadísticas universitarias comienzan a dar cuenta de este dato, hay un crecimiento del número de doctores docentes en las universidades, de casi el $1,5 \%$ del total, es decir unos 2800 docentes universitarios pasan a tener título de doctor en ese período.

Si en 2009 sólo el 8,4\% de los docentes universitarios argentinos eran doctores, ese número sube al $9,9 \%$ en 2013 , y es muy posible siguiendo la proyección, aunque aún no hay estadísticas difundidas, que actualmente esté en torno al $12 \%$.

\begin{tabular}{l|l|l|l|l|l|l} 
() Rev. Inter. Educ. Sup. & Campinas, SP & v.3 & n.1 & p.150-166 & jan./abr. 2017 & ISSN 2446-9424 \\
\hline
\end{tabular}




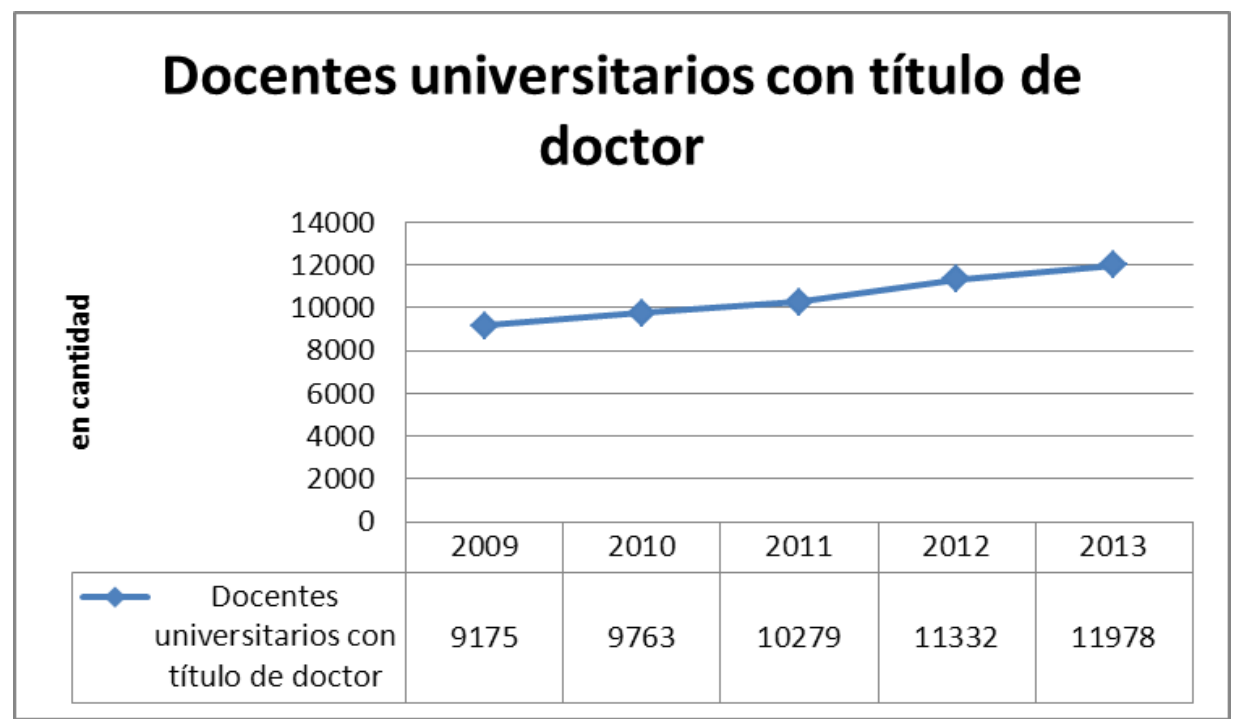

Grafica 7. Docentes universitarios con título de doctor en cantidad (2009-2013) Fuente: Elaboración propia en base a datos de la SPU

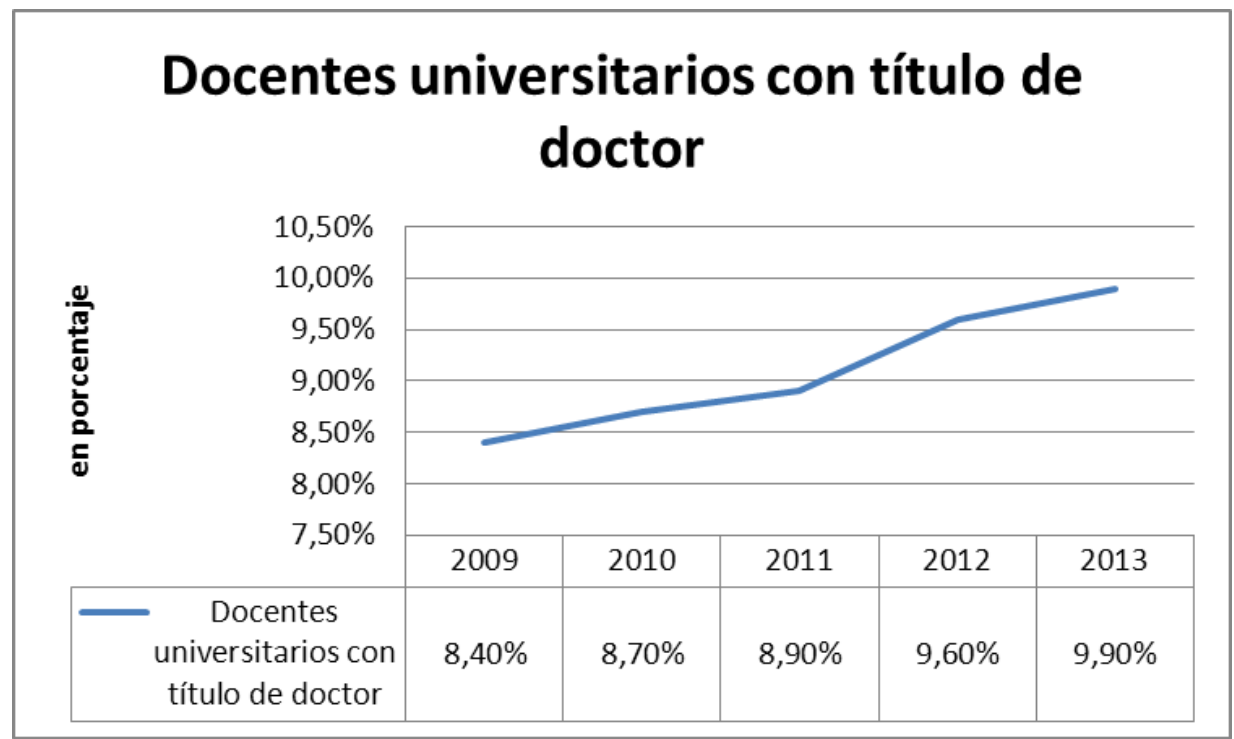

Grafico 8. Docentes universitarios con título de doctor en porcentaje (2009-2013) Fuente: Elaboración propia en base a datos de la SPU

El análisis de esos datos por institución, si bien muestra que en todas las casas de estudio los doctores son absoluta minoría, también reflejaba fuertes dispersiones. Universidades como las Nacional del Sur, Nacional de San Luis, Nacional de Córdoba y Nacional de Río Cuarto comienzan el período por encima del 15\% y siguen creciendo, superando varias el 20\% de sus plantas de docentes con doctorado en 2013.

La contracara son universidades de regiones más periféricas o de reciente creación, como las del Chaco Austral, Formosa o La Rioja que no llegan al 3\% de sus planteles docentes con doctorados en 2013. 
Si tomamos el período 2009-2013, el crecimiento del número de doctores docentes en el sistema universitario argentino es de unos 700 por año, mientras que el incremento del número de doctores en el país en ese lapso fue en promedio de casi 1650 doctores por año.

Esto supone que el $40 \%$ del crecimiento del número de nuevos doctores que se forman en el país se traduce en incrementos en los docentes doctores.

Esos números son preocupantes si ponemos el acento en el modesto rol de las universidades como salida laboral para los doctores, lo que nos remite al ya tradicional problema del predominio de las dedicaciones parciales en la universidad argentina.

Si tomamos el período 2008-2013 (último año procesado) podemos ver que se ha producido un ligero aumento del número de docentes con dedicaciones exclusivas en las universidades nacionales, aunque la participación se ha reducido.

Si en 2008 el 19,5\% de los docentes universitarios tenían dedicación exclusiva, ese número baja al 18,6\% en 2013 y lo mismo pasa con las dedicaciones semi-exclusivas, que pasan del $30 \%$ al $28,3 \%$ en el mismo lapso, tal como podemos apreciar en el siguiente grafico.

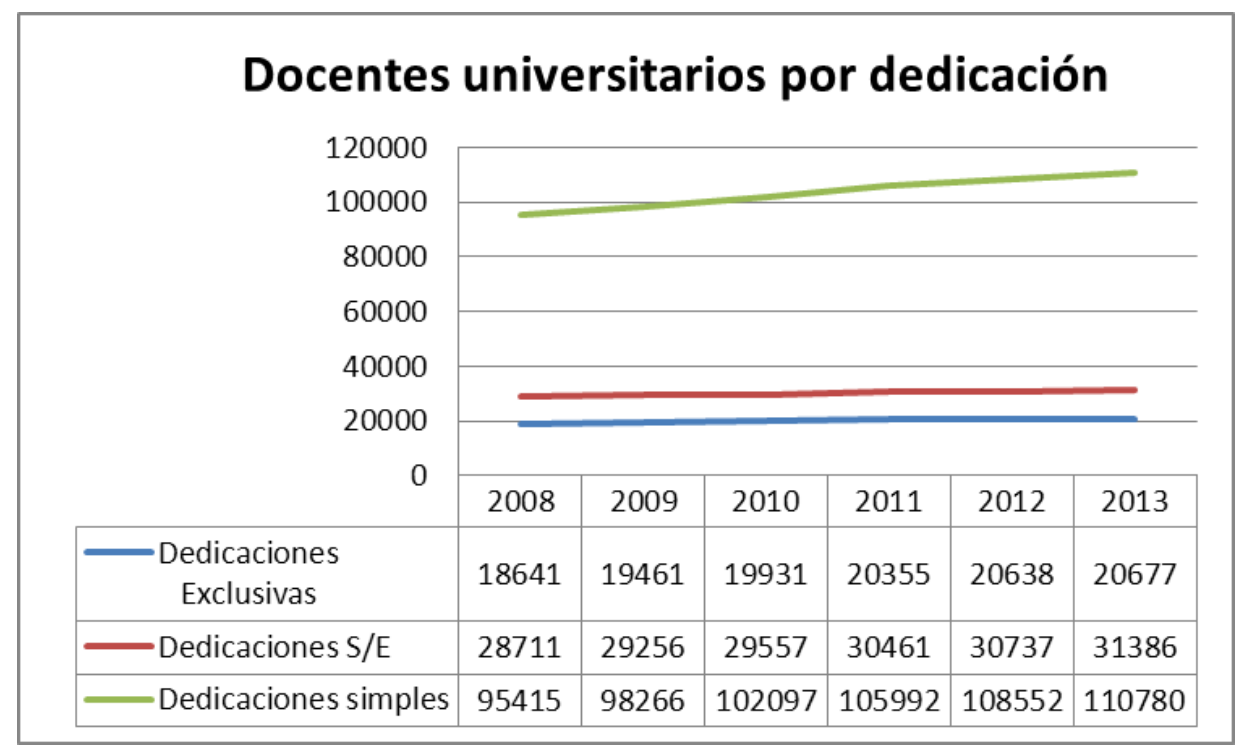

Grafica 9. Dedicaciones de docentes universitarios argentinos 2008-2013 Fuente: Elaboración propia en base a datos de la SPU

Se trata de un punto muy relevante que muestra que el perfil de docencia profesionalista con dedicaciones parciales no ha hecho más que acentuarse en el período, en parte por el proceso de surgimiento de nuevas universidades nacionales que ha sido muy significativo entre los años 2003 y 2015.

El cruce de datos entre dedicaciones y posesión de un doctorado sí muestra que los doctores tienen mayor participación entre los docentes con dedicaciones exclusivas, pero no así en los de dedicaciones semi-exclusivas o simples, donde la participación de los doctores está por debajo de la media mencionada. 
Si el 9,9\% del total de los docentes universitarios son doctores en 2013, ese número llega al 26,6\% de los docentes con dedicación exclusiva, pero es muy inferior en aquellos con dedicaciones semi-exclusivas o simples, donde encontramos respectivamente un $7,3 \%$ y $5,4 \%$.

El cruce por cargo también es un dato relevante, pues sólo el 19,4\% de los profesores titulares serían doctores en 2013, el 21,7\% de los asociados, el 12,1\% de los adjuntos, el 6,6\% de los Jefes de Trabajos Prácticos y el 3\% de los ayudantes de primera.

Vale decir, en todas las categorías del escalafón docente los doctores son minoría, aunque esto podría matizarse si realizamos un análisis por disciplina.

Como parte de una política errática sobre este tema, mientras que en el terreno de las políticas de CyT se decidió promover la formación de doctores con la consecuente inversión en becas, la inserción laboral de los mismos ha sido menos clara.

Superado un primer momento de inserción en algunos organismos de ciencia, su absorción por parte del sistema universitario, tanto público como privado ha sido menos significativa.

La estructura del sistema profesionalista, concentrado en dedicaciones parciales, se ha profundizado, lo que dificulta el despliegue de carreras académicas en el ámbito universitario. Sobre este punto, una excepción puede encontrarse en las regiones más densas en instituciones universitarias, o con universidades de mayor tamaño, que permiten que un mismo agente concentre varias dedicaciones parciales a la docencia, simulando una dedicación completa.

Pero se debe señalar que no se ha logrado introducir elementos que incentiven a las universidades a promover la contratación de doctores, y tampoco se logró aumentar la dedicación de los cargos docentes para profundizar las políticas de investigación de las universidades.

Un matiz no menor, ha sido la incorporación de un adicional salarial por título de doctor, de un monto no despreciable del $15 \%$, que comenzó a implementarse desde el año 2008 .

La génesis de esa innovación fue la discusión salarial con los gremios universitarios (de hecho, posteriormente se introducen otros estímulos salariales a los títulos de maestría y especialización) más que una clara decisión de alterar el perfil de la docencia universitaria argentina.

Este adicional ha operado como un estímulo a los docentes para que emprendan sus doctorados (o los terminen aquellos que los habían iniciado). Los resultados de este último lustro no han sido adecuadamente estudiados hasta el momento. De hecho, ciertas limitaciones presupuestarias parecen estar entorpeciendo el acceso a esos adicionales salariales por título de posgrado, lo que puede poner en juego la eficacia de la decisión en la alteración de la formación de los docentes.

Lo que sí está claro es que el incentivo no es para las universidades, ni lleva a que las universidades sean las que se interesen en contratar doctores, sino más bien, parece operar

\begin{tabular}{l|l|l|l|l|l|l} 
() Rev. Inter. Educ. Sup. & Campinas, SP & v.3 & n.1 & p.150-166 & jan./abr. 2017 & ISSN 2446-9424
\end{tabular}


sobre los docentes ya en posesión de sus cargos, incentivándolos para formarse logrando de ese modo mejoras salariales.

\section{CONCLUSIONES}

El proceso reciente de crecimiento de los doctorados en Argentina ha sido muy relevante desde diversos puntos de vista. Sin embargo el mismo se expresa con mucha mayor claridad en las regiones centrales del país y en algunas áreas disciplinares.

Esto ha sido consecuencia de una política de inversión en la formación de recursos humanos en ciencia y tecnología, que tuvo su expresión más clara a partir del año 2003 y 2004 y sus resultados desde el 2009-2010.

El destino laboral de esos doctores fue esencialmente el sistema científico público argentino, y particularmente su organismo más emblemático: el CONICET, al menos hasta el año 2010, en el que comienza a plantearse cierta tendencia al agotamiento de esa vía.

Notemos que en 2014 el país alcanza la meta de 3 investigadores equivalentes a jornada completa por cada 1000 integrantes de la población económicamente activa.

A pesar de ello, la incidencia de los doctores en los cuerpos de profesores de las universidades argentinas sigue siendo atípicamente baja en términos internacionales.

El crecimiento del número de doctores no ha sido aún capaz de tener incidencia significativa sobre los perfiles predominantes en los cuerpos de profesores universitarios, y no hay evidencia clara de que la situación pueda revertirse en el mediano plazo.

La introducción de la política de adicionales salariales para docentes con títulos de doctor, parece tener cierto efecto dinamizador, aunque sus dificultades actuales pueden llevar a la disolución de ese incentivo.

En todo caso, es esperable que el sistema universitario se siga diferenciando, y que algunas universidades en general más dinámicas en su funcionamiento, ubicadas en regiones centrales, con sesgos menos profesionalistas y con estructuras académicas más volcadas a dedicaciones completas, sean las que logren mejores resultados en el incremento de la integración de sus cuerpos docentes con doctores.

\section{REFERENCIAS}

BARSKY, Osvaldo. Los posgrados universitarios en la República Argentina. Buenos Aires: Troquel, 1997.

CONICET. Eficacia del programa de becas de postgrado del Conicet en la obtención de títulos de doctorado. Buenos Aires, nov. 2016. 
CONICET. Eficacia del programa de becas de postgrado del Conicet en la obtención de títulos de doctorado. Buenos Aires, abr. 2014. Disponible en: <http://www.conicet.gov.ar /wp-content/uploads/2014/05/Informe-de-Eficacia-de-Becas-CONICET-2014.pdf > . Acceso: 10 feb. 2017.

DE LA FARE, Mónica; LENZ, Silvia. El posgrado en el campo universitario: expansión de carreras y productividad de tesis en la Argentina. Buenos Aires: IEC-UNGS, 2012.

FACULTAD DE CIENCIAS EXACTAS DE LA UBA. Informe de gestión 2010-2014. Buenos Aires: UBA, 2014.

FACULTAD DE CIENCIAS SOCIALES DE LA UBA. Secretaría de Estudios Avanzados, Subsecretaría de Posgrado. Composición del perfil de graduados del doctorado de la Universidad de Buenos Aires en Ciencias Sociales 1999-2015. Buenos Aires: UBA, 2015.

ARGENTINA. Ley de Educación Superior n.521, 1995. Disponible en: <https://www.educ.ar/recursos/91820/ley-de-educacion-superior $>$. Acceso: 10 feb. 2017.

LVOVICH, Daniel. Resultados e impactos de los programas de apoyo a la formación de posgrado en Argentina. Revista Iberoamericana CTS, v.5, n.13, nov. 2009. Disponible en: http://www.scielo.org.ar/scielo.php?script=sci_arttext\&pid=S1850-00132009000200009 . Acceso: 15 mar. 2017.

SECRETARÍA DE POLÍTICAS UNIVERSITARIAS. Ministerio de Educación y Deportes. Anuarios de Estadísticas Universitarias. Disponibles en: <http://portales.educacion. gov.ar/spu/investigacion-y-estadisticas/anuarios/>. Acceso: 10 feb. 2017.

UNZUÉ, Martín. Claroscuros del desarrollo de los posgrados en Argentina. Revista Sociedad, Buenos Aires, n. 29-30, 2011. Disponible en: <http://www.sociales.uba.ar/wpcontent/uploads/Sociedad29-30-FINAL2.pdf >. Acceso: 20 feb. 2017. 